\title{
Is continuous positive airway pressure necessarily an everyday therapy in patients with obstructive sleep apnoea?
}

\author{
Valentina A. Rossi ${ }^{1}$, Esther I. Schwarz' ${ }^{1}$ Konrad E. Bloch ${ }^{1,2}$, John R. Stradling ${ }^{3}$ \\ and Malcolm Kohler ${ }^{1,2}$
}

Affiliations: ${ }^{1}$ Sleep Disorders Centre and Pulmonary Division, University Hospital of Zurich, Zurich, and ${ }^{2}$ Zurich Centre for Integrative Human Physiology, University of Zurich, Zurich, Switzerland. ${ }^{3}$ Oxford Centre for Respiratory Medicine, Churchill Hospital and Biomedical Research Centre, Oxford, UK.

Correspondence: M. Kohler, Chair Respiratory Medicine, Clinical Director Division of Pulmonology, University Hospital Zurich, Raemistrasse 100, 8091 Zurich, Switzerland. E-mail: Malcolm.KohlerQusz.ch

ABSTRACT There are limited data on the evolution of obstructive sleep apnoea (OSA) during continuous positive airway pressure (CPAP) therapy and whether this treatment is required every night.

125 OSA patients with an original oxygen desaturation index (ODI) $>10$ events per hour, established on CPAP, were asked to withdraw CPAP for four nights and performed ambulatory nocturnal pulse oximetry on the fourth night of CPAP withdrawal. An ODI $>10$ events per hour during pulse oximetry was considered to indicate persistent OSA. Patients not experiencing recurrence of OSA underwent repeat ambulatory pulse oximetry after a further 2-week period off CPAP.

In $71 \%$ of the patients, OSA recurred after four nights of CPAP withdrawal (group 1); thus, OSA did not recur in 29\% (group 2). 55\% of group 2 had an ODI $>10$ events per hour after 2 weeks off CPAP; thus, $45 \%$ remained without a recurrence. In multivariate analysis, higher original ODI, longer duration of CPAP therapy, current smoking status and larger neck circumference were independently associated with a higher ODI after four nights of CPAP withdrawal (all $\mathrm{p}<0.05$ ).

Following CPAP withdrawal, a third of CPAP-treated patients do not experience significant recurrence of oxygen desaturations after 4 days and $\sim 10 \%$ do not after 2 weeks. Thus, a significant proportion of patients may be able to stop CPAP for short periods.

@ERSpublications

OSA does not recur in one-third of patients after 4 nights of CPAP withdrawal and in $\sim 10 \%$ after 2 weeks http://ow.ly/sxqIl

For editorial comments see page 1227.

Received: Oct 162013 | Accepted after revision: Dec 172013 | First published online: Jan 312014

Support statement: This work was supported by the Swiss National Science Foundation (32003B_143365/1) and the Clinical Research Priority Program Sleep and Health of the University of Zurich.

Conflict of interest: None declared.

Copyright @ERS 2014 


\section{Introduction}

Obstructive sleep apnoea (OSA) is a common sleep-related breathing disorder, which is characterised by transient interruption of ventilation during sleep caused by complete or partial collapse of the upper airway. The condition has been shown to be more prevalent in male subjects, especially in the obese [1,2] with pronounced waist, neck and lingual fat distribution [3, 4]. However, there is a substantial number of patients with OSA in whom these features are not present and, thus, it is not always clear why some people suffer from OSA.

Studies looking at the night-to-night variability of polysomnographic indices showed that, in patients with newly diagnosed OSA, measures of OSA severity such as the apnoea-hypopnoea index (AHI) varied by more than 10 events per hour between two consecutive nights in $32 \%$ of all cases [5]. Furthermore, $22 \%$ of the studied subjects were only diagnosed with OSA during a second sleep study [6]. The reasons underlying this considerable night-to-night variability are largely unknown. Reversible factors such as smoking and alcohol usage, upper airway congestion, oedema of the pharyngeal mucosa, muscle fatigue or hormonal status have been proposed to account for the night-to-night variability [4].

As continuous positive airway pressure (CPAP) therapy has been shown to reduce soft tissue oedema in the pharynx, we hypothesised that this treatment may improve the patency of the upper airways, thus leading to a reduction in OSA recurrence following CPAP withdrawal [7]. As OSA is considered a chronic and mostly persistent condition in subjects who do not lose a substantial amount of weight, CPAP is assumed to be a lifelong and everyday treatment. However, there are no data on the evolution of OSA during CPAP therapy, and whether this treatment needs to be used every night in all patients. Therefore, the aim of the current study was a preliminary investigation into the effects of short-term CPAP withdrawal in patients with OSA and to identify those patients in whom OSA does not recur during short periods, equivalent to the average holiday.

\section{Methods}

Patients

The data of this study were collected during two randomised controlled CPAP withdrawal trials [8, 9]. In both trials, patients included had been previously diagnosed with OSA by in-hospital respiratory polygraphy and treated with CPAP. They were registered in the databases of the Sleep Disorders Centre and Pulmonary Division of the University Hospital Zurich, Zurich, Switzerland, and of the Centre for Respiratory Medicine, Oxford, UK. Apnoeas were defined as a reduction in amplitude of the nasal flow or chest wall motion by $>90 \%$ from baseline over the previous $2 \mathrm{~min}$ for $>10 \mathrm{~s}$; hypopnoeas were defined as a reduction in amplitude of the nasal flow or chest wall motion by $>50 \%$ from baseline over the previous 2 min for $>10 \mathrm{~s}$, associated with $\mathrm{a} \geqslant 4 \%$ drop in oxygen saturation. Patients were eligible for the trial if they were aged between 20 and 75 years, had an oxygen desaturation index (ODI) ( $\geqslant 4 \%$ dips) of $>10$ events per hour during their original diagnostic sleep study and they had been treated with CPAP for $>12$ months with an average compliance of $\geqslant 4 \mathrm{~h}$ per night.

Patients with previous ventilatory failure, Cheyne-Stokes breathing, unstable and untreated coronary or peripheral artery disease, severe and inadequately controlled arterial hypertension, or a history of any sleep related accident, or who were current professional drivers, were excluded from the studies.

\section{Study design}

All patients underwent ambulatory nocturnal pulse oximetry (Pulsox-300i; Konica Minolta Sensing Inc., Osaka, Japan) on the last night of a four-night period without CPAP. This oximeter has a sample frequency of $1 \mathrm{~Hz}$, an averaging time of $3 \mathrm{~s}$ and a resolution of $0.1 \%$ saturation. Patients with an ODI $\leqslant 10$ events per hour after four nights off CPAP were asked to further withdraw CPAP for a 2 -week period. At the end of the 2-week period without CPAP, patients repeated ambulatory home pulse oximetry.

\section{Subjective sleepiness}

Subjective sleepiness was assessed by the Epworth Sleepiness Score (ESS) at baseline and after 2 weeks of CPAP withdrawal $[10,11]$.

\section{OSA recurrence}

The primary outcome of interest of the study was the prevalence of the recurrence of OSA assessed by pulse oximetry after four nights and after 2 weeks of CPAP withdrawal, in patients who have been successfully treated with CPAP for $\geqslant 12$ months. OSA recurrence was defined as an ODI of $>10$ events per hour $(\geqslant 4 \%$ dips $)$. 


\section{Statistical analysis}

All values are presented as mean \pm SD unless otherwise stated. Statistical analysis was performed with SPSS Statistics (version 20.0; IBM, Armonk, NY, USA). Differences in baseline characteristics between groups were assessed by independent t-tests and Chi-squared tests as appropriate. Comparisons of changes within groups were assessed by dependent t-tests.

Multivariate regression analysis was performed to estimate associations among age, original ODI, years of CPAP therapy, neck circumference, body mass index (BMI) and smoking status as independent variables, with ODI after four nights of CPAP withdrawal as the dependent variable. As the number of patients with ODI data after 2 weeks of CPAP withdrawal was relatively small, ODI at 2 weeks was not included in the multivariate analysis. A p-value of $<0.05$ was considered to indicate statistical significance.

\section{Results}

\section{Study population}

125 patients were enrolled in the study. The study population was divided into two groups based on the ODI after four nights of CPAP withdrawal. Group 1 (ODI $>10$ events per hour) included 89 patients; group 2 (ODI $\leqslant 10$ events per hour) included 36 patients.

Table 1 summarises the characteristics of the two groups. Patients in group 1 were more obese and had a greater neck circumference than patients in group 2. Group 2 presented lower values of AHI at diagnosis, as well as while on CPAP treatment, and had been treated for a shorter period of time than group 1. Successful treatment of patients with CPAP prior to the study was confirmed from data on AHI downloaded from the patients' CPAP machines, as well as ESS values within the normal range (table 1). $34 \%$ of the patients in group 2 met the criteria for severe OSA (ODI $>30$ events per hour) at the time of diagnosis, compared with $47 \%$ in group 1.

Of the 36 patients in group 2, 23 agreed to withdraw CPAP therapy for 2 weeks and underwent a second ambulatory pulse oximetry. Among these, three patients withdrew due to increased sleepiness during the CPAP withdrawal phase. Figure 1 shows the patient flow.

\section{OSA recurrence}

In $89(71 \%)$ out of 125 patients, OSA recurred after four nights of CPAP withdrawal (group 1), thus in 36 (29\%) out of 125, OSA did not recur (group 2). 20 out of this 36 agreed to the second study and 11 (55\%) had an ODI $>10$ events per hour after 2 weeks off CPAP; thus, nine (45\%) out of 20 in group 2 remained without a recurrence of OSA (figs 1 and 2).

Assuming those who declined the second study were no different to the 20 patients who accepted, then a total of $16(13 \%)$ out of the original 125 would have had no return of OSA. Assuming all those who

TABLE 1 Characteristics of the two groups

\begin{tabular}{|c|c|c|c|}
\hline & Group $1^{\#}$ & Group 2 & p-value \\
\hline Subjects $n$ & 89 & 36 & \\
\hline Age years & $63 \pm 9.2$ & $60 \pm 10$ & 0.111 \\
\hline Males/females $n$ & $75 / 14$ & $30 / 6$ & 0.774 \\
\hline Body mass index at diagnosis $\mathrm{kg} \cdot \mathrm{m}^{-2}$ & $34.2 \pm 8.8^{f}$ & $27.7 \pm 4.5^{\# \#}$ & 0.024 \\
\hline Body mass index at time of study $\mathrm{kg} \cdot \mathrm{m}^{-2}$ & $34.0 \pm 6.2$ & $30.1 \pm 4.9$ & 0.001 \\
\hline Neck circumference $\mathrm{cm}$ & $44.0 \pm 4.0$ & $41.7 \pm 2.7$ & $<0.001$ \\
\hline Current smokers $\%$ & 12 & 22 & 0.497 \\
\hline $\mathrm{AHI}$ in original sleep study events per hour & $38.6 \pm 21.1$ & $28.4 \pm 12.3$ & 0.008 \\
\hline ODI in original sleep study events per hour & $30.9 \pm 14.5$ & $26.6 \pm 10.1$ & 0.112 \\
\hline $\mathrm{AHI}$ on $\mathrm{CPAP}^{+}$events per hour & $4.8 \pm 3.3$ & $3.4 \pm 2.8$ & 0.024 \\
\hline CPAP compliance $^{\S} \mathrm{h}$ per night & $6.0 \pm 1.5$ & $6.0 \pm 1.5$ & 0.200 \\
\hline ESS before therapy & $14.5 \pm 3.2$ & $15.1 \pm 4.0$ & 0.410 \\
\hline ESS on CPAP & $6.8 \pm 2.4$ & $6.4 \pm 2.6$ & 0.436 \\
\hline Duration of CPAP treatment years & $6.5 \pm 4.4$ & $4.6 \pm 3.4$ & 0.045 \\
\hline
\end{tabular}

Data are presented as mean \pm SD, unless otherwise stated. AHI: apnoea-hypopnoea index; ODI: oxygen desaturation index; CPAP: continuous positive airway pressure; ESS: Epworth Sleepiness Score; ${ }^{\#}$ : ODI $>10$ events per hour; ${ }^{\top}$ : ODI $\leqslant 10$ events per hour; ${ }^{+}$: downloaded from CPAP machine, averaged from previous 6 months; ${ }^{\S}$ : derived from CPAP machine; ${ }^{f}: n=34 ; \# \#: n=11$. 


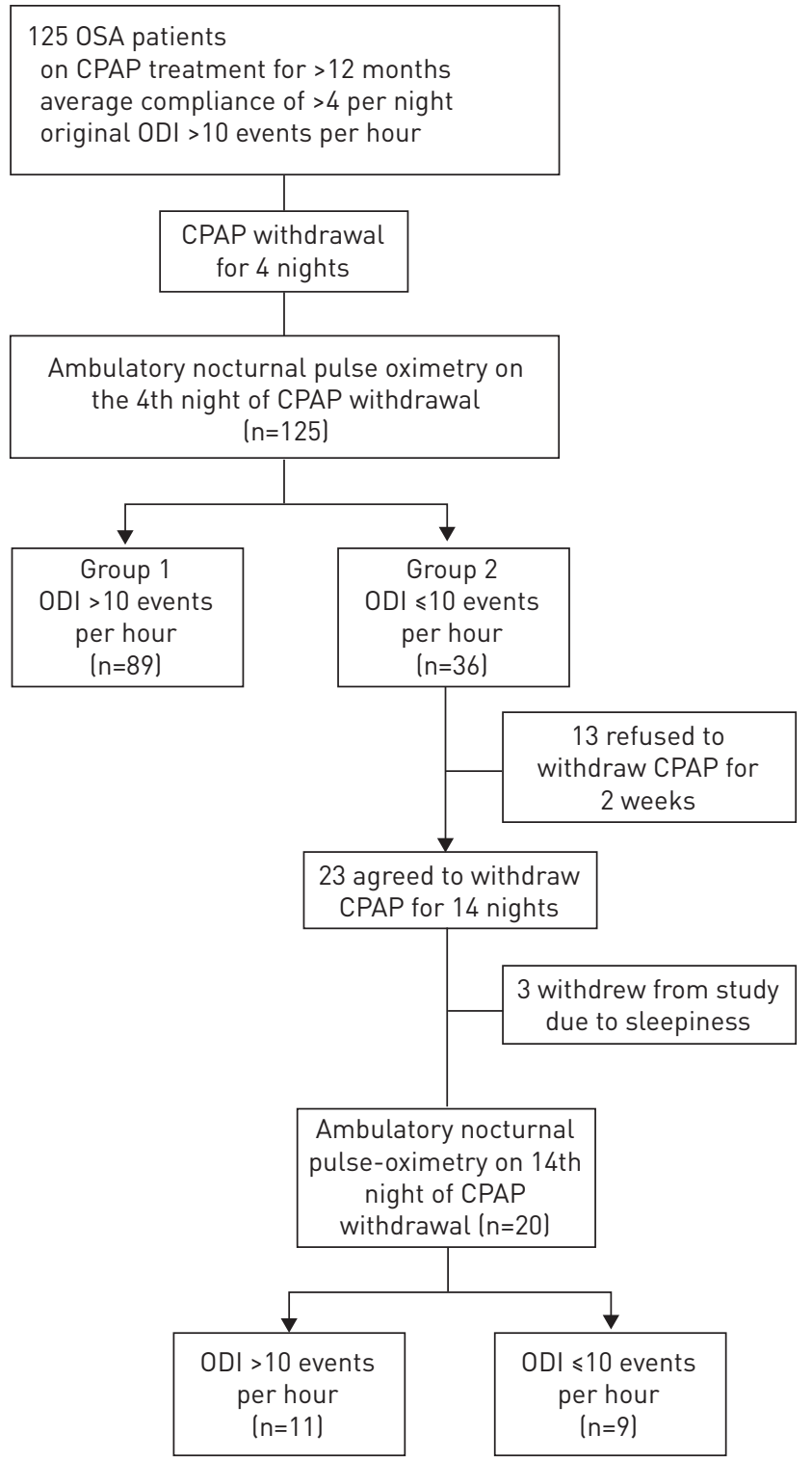

FIGURE 1 Patient flow. OSA: obstructive sleep apnoea; CPAP: continuous positive airway pressure; ODI: oxygen desaturation index.

declined the second study had a return of OSA, then nine (7\%) out of the original 125 would have had no return of OSA. This gives a range of $7-13 \%$ of patients who do not relapse following 14 nights off CPAP.

Based on the original sleep study at diagnosis, and using conventional thresholds for defining OSA severity, $11.3 \%$ suffered from mild OSA (ODI 5-15 events per hour), 46.8\% from moderate (ODI 15-30 events per hour) and $41.9 \%$ from severe OSA (ODI $>30$ events per hour). After four nights of CPAP withdrawal, $16 \%$ showed no recurrence of OSA (ODI $<5$ events per hour), and $28.8 \%$ had mild, $29.6 \%$ moderate and $25.6 \%$ severe OSA.

\section{Subjective sleepiness}

Patients in group 2 who underwent 2 weeks of CPAP withdrawal had a statistically significantly higher ESS after the 2 weeks, compared with their previous ESS on CPAP therapy $(10.1 \pm 5.1$ versus $6.4 \pm 2.6, \mathrm{p}=0.003)$. However, there was no statistically significant difference in ESS between patients in whom OSA recurred at 2 weeks, compared to those who did not relapse $(8.5 \pm 5.9$ versus $12.1 \pm 3.2, \mathrm{p}=0.11)$.

\section{Multivariate analysis}

Multivariate analysis included age, original ODI, years of CPAP therapy, neck circumference, BMI and current smoking status as independent variables, and ODI after four nights of CPAP withdrawal as the dependent variable. Higher original ODI $(\beta \pm$ SE $0.22 \pm 0.11, p=0.02)$, longer duration of CPAP therapy 

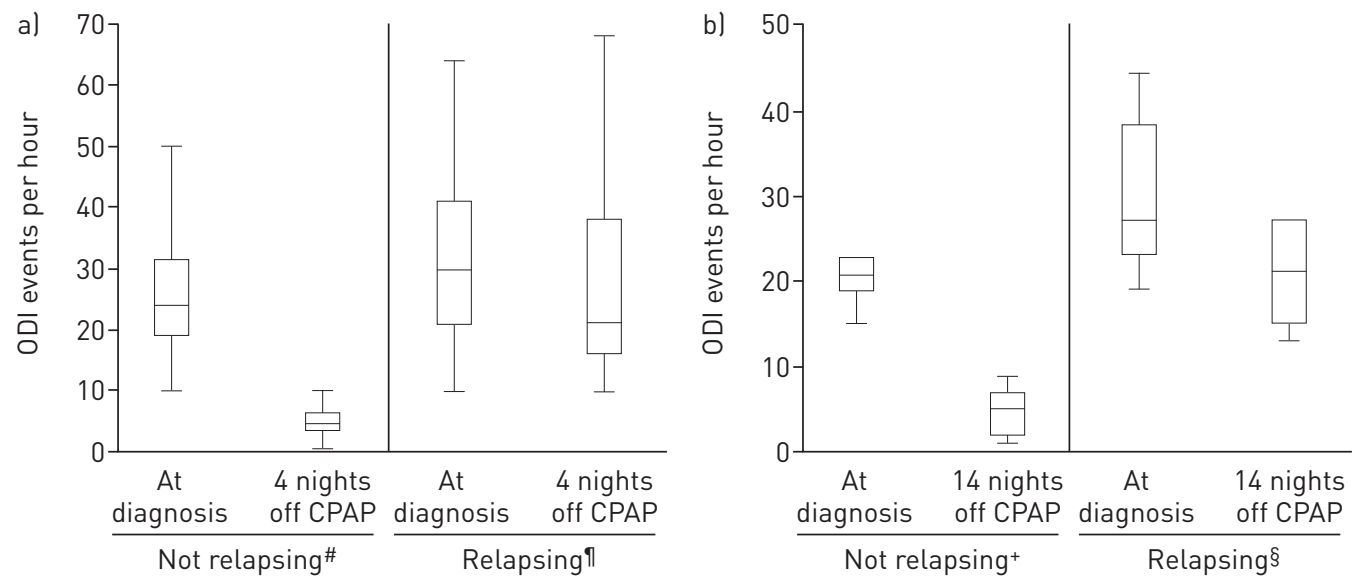

FIGURE 2 a) Oxygen desaturation index (ODI) at the time of the original diagnosis and during the fourth night off continuous positive airway pressure (CPAP) in patients without obstructive sleep apnoea (OSA) relapse and patients with OSA relapse. b) In the subset of patients who did not have a relapse of OSA after four nights of CPAP withdrawal, a further 2-week CPAP withdrawal was performed. The ODI at the time of the original diagnosis and during the 14th night off CPAP in patients without OSA relapse and patients with OSA relapse is shown. ${ }^{\#}: n=36 ;{ }^{\uparrow}: n=89 ;{ }^{+}: n=9{ }^{\S}: n=11$.

( $\beta$ 0.36, SE 0.38; $\mathrm{p}<0.001)$, larger neck circumference $(\beta 0.24$, SE $0.43 ; \mathrm{p}=0.02)$ and current smoking status ( $\beta$ 0.21, SE 3.0; $p=0.027$ ) were independently associated with a higher ODI after CPAP withdrawal. There was no statistically significant association with age $(\beta-0.16$, SE $0.16 ; p=0.87)$ or BMI $(\beta 0.11$, SE 0.27 ; $\mathrm{p}=0.28$ ). However, only $34 \%$ of the variation in ODI after four nights of CPAP withdrawal was explained by the model including all variables $\left(\mathrm{r}^{2}=0.344\right)$.

\section{Discussion}

We have shown that a third of patients with prior moderate-to-severe OSA can stop their CPAP treatment for four nights without a significant return of OSA, and nearly half of these did not have a return of OSA even after 2 weeks off CPAP. In general, patients with less severe OSA originally, smaller neck circumferences or shorter time on CPAP, and current non-smokers were those less likely to have a return of OSA after four nights off CPAP. However, this was not always the case, and the predictive ability of these factors would be of limited use in clinical practice in determining who might do well off CPAP for short periods. We cannot, of course, be sure that this nonrecurrence of OSA in some patients was due to the period on CPAP itself, as it might have occurred spontaneously even if the patients had not received CPAP.

In a recent uncontrolled study, two nights of CPAP therapy withdrawal led to lower AHI values, with less severe oxygen desaturations, compared to pre-treatment values, in 42 patients with severe sleep-disordered breathing and prior good adherence to CPAP treatment [12]. In a randomised controlled trial of 41 subjects, CPAP withdrawal for 2 weeks was associated with full return of OSA within the first week in nearly all subjects, versus control subjects who remained on CPAP, with statistical significance already achieved on the first night off CPAP [8]. However, prior to entry into this study, the patients had already demonstrated a recurrence of OSA after four nights off CPAP, and those who did not relapse had already been excluded. In a study looking at modafinil as an aid to preserving vigilance, two nights of CPAP withdrawal also led to rapid but variable return of OSA [13], and in an earlier uncontrolled study, the same group of authors showed increasing OSA return after one and seven nights of treatment withdrawal in 20 CPAP-compliant subjects with prior moderate-to-severe OSA [14]. Again, the severity of the return was variable and the mean respiratory disturbance index for the group, even after seven nights' withdrawal, did not reach the original diagnostic sleep study values. An earlier uncontrolled study looking at one night of CPAP withdrawal in 30 patients with severe OSA and good compliance for a year following diagnosis and treatment also showed variable and partial return of OSA [15]. Thus, all prior studies showed that OSA did not necessarily return after CPAP withdrawal for up to seven nights, and our current study has shown this to be true for up to 2 weeks as well.

It is unclear, in the absence of weight loss, why OSA should appear to be "cured", at least temporarily. In all studies looking at causes of OSA, the factors identified (usually neck size, craniofacial shape, tongue size and abdominal obesity) rarely explain $>50 \%$ of the variation in OSA severity. This implies there are other factors, some of which may fluctuate with time. Any condition that has a natural fluctuation in its severity will tend to present when at its worst, and when restudied at a later date tend, on average, to be better, i.e. regression to the mean. The same phenomenon, of course, also applies to the actual diagnostic sleep study: 
the higher initial ODI or AHI values will tend to be lower on a second study. It is unclear what might generate fluctuations in severity night-to-night, week-to-week or month-to-month, but alcohol use, upper airway congestion and pharyngeal oedema, pharyngeal dilator muscle efficacy, body position, and hormonal status are a few suggestions.

It is possible that a period on CPAP might reverse and temporarily "cure" some of these factors. RYAN et al. [7], in 1991, performed pharyngeal magnetic resonance imaging scans on five patients with OSA before and after 4-6 weeks of CPAP. They showed a reduction in pharyngeal oedema and tongue size, leading to an increase in pharyngeal volume [7]. Presumably, the pre-CPAP oedema resulted from pharyngeal trauma caused by snoring and repetitive apnoeas, and its resolution might make the difference between having significant OSA or not. It is also possible that this reduction in oedema might improve reflex pharyngeal dilator muscle activity in response to improved mucosal surface receptor activity [16, 17]. Others have suggested that increased pharyngeal dilator muscle activity might lead to a degree of chronic muscle fatigue [18] that could be reversible following a period on CPAP. These causes of a temporary cure might then hold for a variable period of time until OSA begins to return perhaps, for example, following a period of increased alcohol intake, an upper respiratory tract infection or enforced supine sleeping (following an operation, for example); it would then require further CPAP therapy to reverse the damage done.

This variable return of OSA following CPAP withdrawal, as measured by ODI, suggests that perhaps sleep physicians could be suggesting that patients try coming off CPAP from time-to-time to establish if they still need to use it every night; this could be with or without the addition of more convenient therapies, such as mandibular advancement devices [19], that might not work initially but could do so after a period on CPAP. It might then be possible to identify a patient's particular precipitants for a return of OSA and thus learn to avoid them. There are many other diseases that require increased therapy during exacerbations but not during the quiescent periods in between. Furthermore, just as with peak flow meters and asthma, it may be viable for patient to use home diagnostic devices to monitor whether their OSA is returning, such as oximeters, snoring detectors or nasal flow monitors, along with symptom scores. Clearly, such a continuing approach would only apply to those shown to have OSA that "resolves" after a period on CPAP, and also might not be appropriate for patients in vigilance-critical employment.

There are some limitations to this study. First, patients with sleepiness-related accidents were excluded and a larger number of patients were approached than ultimately consented, initially for the four night withdrawal and subsequently for the 2-week withdrawal. It is likely that the exclusion criteria and the considerable drop-out rate biased our study population towards those who felt they could withdraw without experiencing problems; perhaps based on their own prior experience. The extent of this bias is impossible to assess.

Secondly, pulse oximetry and apnoea/hypopnoea detection may underestimate the degree of the disease in some patients, and we recognise they are not a gold standard with which to detect all the abnormalities resulting from pharyngeal narrowing during sleep; for example, they do not quantify "upper airway resistance syndrome". However, this method is able to detect hypopnoeas (when defined according to American Academy of Sleep Medicine criteria to require an oxygen desaturation) and it has often been argued that intermittent hypoxia is the main driver of many of the physiological consequences of OSA [20]. In addition, most studies comparing ODI and AHI find close agreement [21-23]. Thus, pulse oximetryderived data on changes in oxygen saturation offer valid information for studying the immediate consequences of OSA. Longer term consequences of OSA, such as daytime symptoms and any cardiovascular sequelae, may require other methods of assessment, although ODI has also been shown to be a good predictor of cardiovascular disease [24].

Finally, although OSA by oximetry did not return in some after 2 weeks, the rise in ESS in this group may be due to increased sleep fragmentation, perhaps from upper airway resistance syndrome. Alternatively, the ESS scores could have been biased upwards, as participants were not of course blinded to their lack of therapy [25]. Without further cognitive function testing we do not know the functional significance of this rise in ESS.

This discussion is of course speculative, but the evidence from ours and others' studies of apparent temporary "cure" of OSA in a proportion of patients on CPAP, for up to two weeks, does suggest that there perhaps should be a change from the mind-set that once CPAP is prescribed, it needs to be used forever. This more flexible approach to CPAP usage might even improve overall compliance.

\section{Conclusions}

Following CPAP withdrawal, we found rather surprisingly that almost one third of CPAP-treated patients with moderate-to-severe OSA did not experience significant recurrence of oxygen desaturations after four 
days, and about $10 \%$ did not after two weeks. However, more subtle sleep disruption could have recurred, such as from upper airway resistance syndrome, and be responsible for an increase in daytime sleepiness. If our findings are confirmed, particularly in trials looking at the effects of a longer period off CPAP including cognitive function and cardiovascular outcomes, then routine clinical practice might need to incorporate such checks for continued CPAP requirement. Further studies are also needed to predict which patients in clinical practice can temporarily, or permanently, withdraw CPAP without recurrence of OSA and significant adverse consequences. Whether such patients remain susceptible to subsequent relapse, and what are the precipitants, will also need further investigation.

\section{References}

1 Dixon JB, Dixon ME, Anderson ML, et al. Daytime sleepiness in the obese: not as simple as obstructive sleep apnea. Obesity 2007; 15: 2504-2511.

2 Young T, Palta M, Dempsey J, et al. The occurrence of sleep-disordered breathing among middle-aged adults. N Engl J Med 1993; 328: 1230-1235.

3 Davidson T, Patel M. Waist circumference and sleep disordered breathing. Laryngoscope 2008; 118: 339-347.

4 Lin CM, Davidson TM, Ancoli-Israel S. Gender differences in obstructive sleep apnea and treatment implications. Sleep Med Rev 2008; 12: 481-496.

5 Chediak AD, Acevedo-Crespo JC, Seiden DJ, et al. Nightly variability in the indices of sleep-disordered breathing in men being evaluated for impotence with consecutive night polysomnograms. Sleep 1996; 19: 589-592.

6 Le Bon O, Hoffmann G, Tecco J, et al. Mild to moderate sleep respiratory events: one negative night may not be enough. Chest 2000; 118: 353-359.

7 Ryan CF, Lowe AA, Li D, et al. Magnetic resonance imaging of the upper airway in obstructive sleep apnea before and after chronic nasal continuous positive airway pressure therapy. Am Rev Respir Dis 1991; 144: 939-944.

8 Kohler M, Stoewhas AC, Ayers L, et al. Effects of continuous positive airway pressure therapy withdrawal in patients with obstructive sleep apnea: a randomized controlled trial. Am J Respir Crit Care Med 2011; 184: $1192-1199$.

9 Rossi VA, Winter B, Rahman NM, et al. The effects of Provent on moderate to severe obstructive sleep apnoea during continuous positive airway pressure therapy withdrawal: a randomized controlled trial. Thorax 2013; 68: 854-859.

10 Johns MW. A new method for measuring daytime sleepiness: the Epworth sleepiness scale. Sleep 1991; 14: 540-545.

11 Bloch KE, Schoch OD, Zhang JN, et al. German version of the Epworth Sleepiness Scale. Respiration 1999; 66: 440-447.

12 Young LR, Taxin ZH, Norman RG, et al. Response to CPAP withdrawal in patients with mild versus severe obstrucitve sleep apnea/hypopnea syndrome. Sleep 2013; 36: 405-412.

13 Williams SC, Marshall NS, Kennerson M, et al. Modafinil effects during acute continuous positive airway pressure withdrawal: a randomized crossover double-blind placebo-controlled trial. Am J Respir Crit Care Med 2010; 181: 825-831.

14 Phillips CL, Yang Q, Williams A, et al. The effect of short-term withdrawal from continuous positive airway pressure therapy on sympathetic activity and markers of vascular inflammation in subjects with obstructive sleep apnoea. J Sleep Res 2007; 16: 217-225.

15 Sforza E, Lugaresi E. Daytime sleepiness and nasal continuous positive airway pressure therapy in obstructive sleep apnea syndrome patients: effects on chronic treatment and 1-night therapy withdrawal. Sleep 1995; 18: 195-201.

16 Friberg D, Gazelius B, Hökfelt T, et al. Abnormal afferent nerve endings in the soft palatal mucosa of sleep apnoics and habitual snorers. Regul Pept 1997; 71: 29-36.

17 Daulatzai MA. Role of sensory stimulation in amelioration of obstructive sleep apnea. Sleep Disord 2011; 2011: 596879.

18 McSharry D, O’Connor C, McNicholas T, et al. Genioglossus fatigue in obstructive sleep apnea. Respir Physiol Neurobiol 2012; 183: 59-66.

19 Smith DM, Stradling JR. Can mandibular advancement devices be a satisfactory substitute for short term use in patients on nasal continuous positive airway pressure? Thorax 2002; 57: 305-308.

20 Kohler M, Stradling JR. Mechanisms of vascular damage in obstructive sleep apnea. Nat Rev Cardiol 2010; 7: $677-685$.

21 Vázquez JC, Tsai WH, Flemons WW, et al. Automated analysis of digital oximetry in the diagnosis of obstructive sleep apnoea. Thorax 2000; 55: 302-307.

22 Ng SS, Chan TO, To KW, et al. Validation of a portable recording device (ApneaLink) for identifying patients with suspected obstructive sleep apnoea syndrome. Intern Med J 2009; 39: 757-762.

23 Chung F, Liao P, Elsaid H, et al. Oxygen desaturation index from nocturnal oximetry: a sensitive and specific tool to detect sleep-disordered breathing in surgical patients. Anesth Analg 2012; 114: 993-1000.

24 Punjabi NM, Newman AB, Young TB, et al. Sleep-disordered breathing and cardiovascular disease: an outcomebased definition of hypopneas. Am J Respir Crit Care Med 2008; 177: 1150-1155.

25 Pépin JL, Guillot M, Tamisier R, et al. The upper airway resistance syndrome. Respiration 2012; 83: 559-566. 